\title{
Performance of Multiuser Detection with Adaptive Channel Estimation
}

\author{
Milica Stojanovic, Member, IEEE, and Zoran Zvonar, Senior Member, IEEE
}

\begin{abstract}
An adaptive multipath decorrelating multiuser receiver is considered for application in Rayleigh fading multipath channels with significant Doppler spread. Coherent diversity combining is performed using adaptively obtained channel estimates in a manner that minimizes the impact of estimation errors on data detection. The bit-error rate of the receiver is evaluated analytically, showing dependence on the fading rate of the channel and tracking capabilities of adaptive least mean square and recursive least square algorithms, in addition to the order of multipath diversity and the number of active codedivision multiple-access users.
\end{abstract}

Index Terms - Code-division multiple access, diversity methods, fading channels.

\section{INTRODUCTION}

$\mathbf{L}$ INEAR multiuser detection for frequency-selective Rayleigh fading code-division multiple-access (CDMA) channels is considered. Previous work in this area has addressed the performance of a number of detection schemes on slowly fading channels (e.g., [1]-[3]). For channels whose Doppler spreading cannot be neglected, the performance of multiuser receivers has been treated in [4]-[7]. For the most recent survey of results in this area, see [10].

The receiver of interest to this study uses multipath decorrelation [2] to eliminate multiple-access interference (MAI) prior to multipath combining, which is then performed with the aid of adaptively obtained channel estimates. Maximal-ratio combiner is robust in the sense that its parameters are optimized to take into account the channel estimation errors. We assess the performance in frequency-selective fading channels by evaluating the bit-error rates in terms of channel fading rate, least mean squares (LMS) and recursive least square (RLS) algorithm tracking capabilities, number of active users, and order of multipath diversity. Results demonstrate the fadinginduced performance degradation, which leads to an error probability floor. Performance of coherent reception depends on the receiver's tracking capability, i.e., the deviation of the tracking parameter (step size of the LMS or forgetting factor of the RLS algorithm) from the optimal value.

\section{Performance Evaluation}

Following the model in [2], we consider a synchronous CDMA system in which the transmitted signal of the $k$ th user

Paper approved by U. Mitra, the Editor for Spread Spectrum/Equalization of the IEEE Communications Society. Manuscript received November 15, 1996 revised March 27, 1998 and December 18, 1998. This paper was presented in part at the IEEE Communication Conference (ICC'97), Montreal, Canada, June 1997.

M. Stojanovic is with the Department of Electrical and Computer Engineering, Northeastern University, Boston, MA 02115 USA (e-mail: milica@cdsp.neu.edu).

Z. Zvonar is with the Communications Division, Analog Devices, Wilmington, MA 01887 USA (e-mail: zoran.zvonar@analog.com).

Publisher Item Identifier S 0090-6778(99)06290-X. is represented in an equivalent complex baseband form

$$
u_{k}(t)=\sum_{n} d_{k}(n) s_{k}(t-n T), \quad k=1, \cdots, K
$$

where $d_{k}(n) \in\{ \pm 1\}$ are data symbols, and $s_{k}(t)$ is the $k$ th user's signature waveform. The signals from $K$ users propagate through different fading channels, independent among various users, modeled as tapped delay lines with taps spaced at chip intervals $T_{c}$ and time varying gains [9]. For a Rayleigh fading channel, the $L$ tap gains of the $k$ th user's channel $\left\{c_{k, l}(t)\right\}$ are complex zero-mean Gaussian processes. The multipath spread is assumed to be much shorter than the data symbol duration, so that any intersymbol interference can be neglected; it suffices to observe the received signal during a single symbol interval

$$
v(t)=\sum_{k=1}^{K} \boldsymbol{c}_{k}^{T}(t) s_{k}(t) d_{k}+n(t)
$$

where $c_{k}(t)$ and $s_{k}(t)$ are appropriately defined vectors of channel tap gains and chip-delayed signature waveforms of the $k$ th user, respectively, and $n(t)$ is complex zero-mean AWGN of power spectral density $N_{0}$. This model is not applicable for spectrally efficient CDMA cases with low-spreading ratio analyzed in [3].

The fading channel dynamics are described by a Gauss-Markov model [5], [6]. The time variations of the channel are modeled as symbol-to-symbol changes in discrete-time vectors of channel tap gains $\boldsymbol{c}_{k}^{T}(n T) \equiv \boldsymbol{c}_{k}^{T}(n)$. If the multipath components fade independently, a simplified second-order model is given by $\boldsymbol{c}_{k}(n+1)=$ $f_{0} c_{k}(n)+f_{1} c_{k}(n-1)+\nu_{k}(n)$ where the components of $\nu_{k}(n)$ are independent zero-mean white Gaussian processes, and $f_{0}, f_{1}$ are real-valued model parameters. The statistics of each channel are described by the covariance matrix $\boldsymbol{C}_{k}=E\left\{\boldsymbol{c}_{\boldsymbol{k}_{i}}(n) \boldsymbol{c}_{k_{k}}^{\prime}(n)\right\}$ (prime denotes conjugate transpose), whose diagonal elements represent the sampled multipath intensity profile of the $k$ th user's channel.

The multipath decorrelating receiver, originally proposed in [2], is depicted in Fig. 1. Matched filtering using signature waveforms results in the signal

$$
x=\int_{0}^{T} \boldsymbol{s}^{*}(t) v(t) d t
$$

which lends itself to partial decorrelation using the known matrix $R=\int_{0}^{T} \boldsymbol{s}^{*}(t) \boldsymbol{s}^{T}(t) d t$ of signature waveforms cross correlations. The output of the decorrelator $z=R^{-1} x$ associated with the $k$ th user is the signal $z_{k}=c_{k} d_{k}+\xi_{k}$, where the desired signal terms $\boldsymbol{c}_{k} d_{k}$ do not contain MAI. The noise terms $\xi_{k}$ are zero-mean Gaussian with covariance 


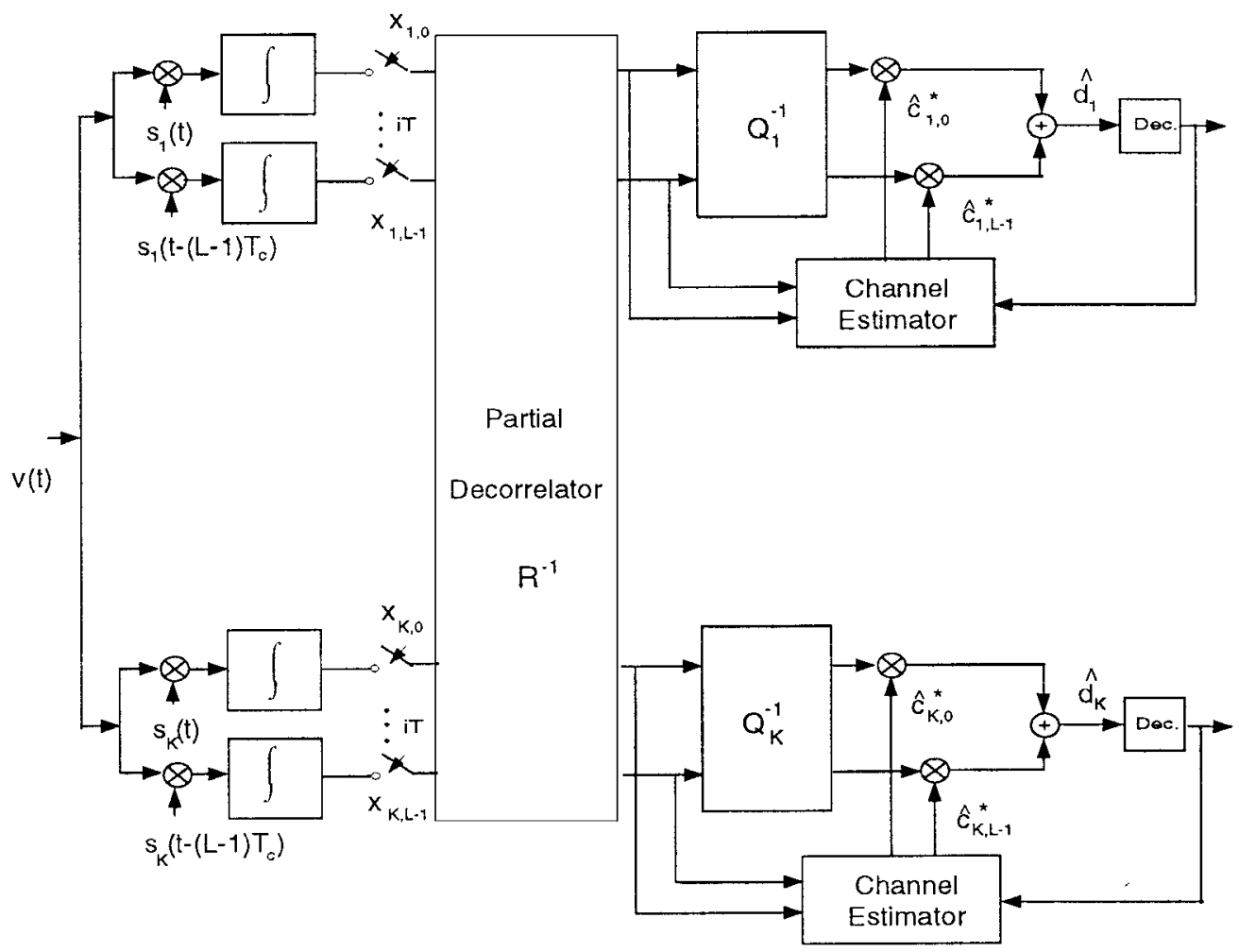

Fig. 1. Adaptive decorrelating receiver.

$\boldsymbol{N}_{k}=\left[N_{0} \boldsymbol{R}^{-1}\right]_{k, k}$ (the $L \times L(k, k)$ th subblock). In the case of long spreading codes, decorrelator is time varying [10].

Having eliminated MAI, the signals $z_{k}$ are processed separately in the remaining part of the receiver. The combiners are optimized subject to the fact that only the channel estimates $\hat{c}_{k}(n)$, and not the true responses, are available. It is assumed that the channel vector $c_{k}(n)$, conditioned on the channel estimate $\hat{\boldsymbol{c}}_{k}(n)$, is a complex Gaussian process with mean $\hat{c}_{k}(n)$ and covariance $\boldsymbol{E}_{k}=E\left\{\boldsymbol{\epsilon}_{k}(n) \boldsymbol{\epsilon}_{k}^{\prime}(n)\right\}$ where $\boldsymbol{\epsilon}_{k}(n)=$ $c_{k}(n)-\hat{c}_{k}(n)$ is the channel estimation error at time $n$. Since the channel vector is Gaussian distributed and estimator is linear, channel vector estimate will also be Gaussian distributed, as well as the estimation error. Maximal-ratio combining results in the estimated data symbol $\hat{d}_{k}(n)=\hat{\boldsymbol{c}}_{k}^{\prime}(n) \boldsymbol{Q}_{k}^{-1} \boldsymbol{z}_{k}(n)$ where $\boldsymbol{Q}_{k}=\boldsymbol{E}_{k}+\boldsymbol{N}_{k}$ is the covariance of the overall noise interfering with the combining process. Assuming that the effect of erroneous bit decisions on channel estimation can be neglected, the lower bound on the $k$ th user's bit error is $P_{k}=P\left\{\operatorname{Re}\left[\hat{d}_{k}(n)\right]<0 \mid d_{k}(n)=+1\right\}=P\left\{G_{k}<0\right\}$ where the variable $G_{k}$ can be expressed as

$$
G_{k}=\boldsymbol{g}_{k}^{\prime}\left[\begin{array}{ll}
\mathbf{0} & \boldsymbol{I} \\
\boldsymbol{I} & \mathbf{0}
\end{array}\right] \boldsymbol{g}_{k}, \boldsymbol{g}_{k}=\left[\begin{array}{c}
Q_{k}^{-1} \hat{c}_{k} \\
\boldsymbol{c}_{k}+\xi_{k}
\end{array}\right]
$$

Under the Rayleigh fading assumption, $G_{k}$ is a Gaussian quadratic form whose distribution is known [9]. The resulting probability $P_{k}$ will depend only on the eigenvalues of the matrix

$$
\boldsymbol{M}_{k}=\left[\begin{array}{cc}
\mathbf{0} & \boldsymbol{I} \\
\boldsymbol{I} & \mathbf{0}
\end{array}\right] E\left\{\boldsymbol{g}_{k} \boldsymbol{g}_{k}^{\prime}\right\}
$$

and we denote this dependence by $P_{k}=P\left[\boldsymbol{M}_{k}\right]$. In particular, when all the eigenvalues $\lambda_{i}$ of $\boldsymbol{M}_{k}$ are distinct

$$
P_{k}=\sum_{i: \lambda_{i}<0} \prod_{j \neq i} \frac{1}{1-\lambda_{j} / \lambda_{i}}
$$

If we denote the cross correlation between the estimation error and the channel vector by $X_{k}=E\left\{\boldsymbol{\epsilon}_{k}(n) \boldsymbol{c}_{k}^{\prime}(n)\right\}$, the matrix $M_{k}$ can be expressed as

$$
\boldsymbol{M}_{k}=\left[\begin{array}{cc}
{\left[\boldsymbol{C}_{k}-\boldsymbol{X}_{k}^{\prime}\right] \boldsymbol{Q}_{k}^{-1}} & C_{k}+N_{k} \\
\boldsymbol{Q}_{k}^{-1}\left[\boldsymbol{C}_{k}-\boldsymbol{X}_{k}-\boldsymbol{X}_{k}^{\prime}+\boldsymbol{E}_{k}\right] \boldsymbol{Q}_{k}^{-1} & \boldsymbol{Q}_{k}^{-1}\left[\boldsymbol{C}_{k}-\boldsymbol{X}_{k}\right]
\end{array}\right] .
$$

The channel estimation error covariances depend on the particular estimation method used. In contrast to Kalman filtering [7], an LMS channel estimator is not model-based. Its estimation error covariance will depend on the value of the step size in addition to the channel parameters. An LMS channel estimator, which uses as an input the data symbols $d(n)$, is defined by the recursion (in the following, we drop the user index for simplicity)

$$
\begin{aligned}
\hat{\boldsymbol{z}}(n) & =\hat{\boldsymbol{c}}(n) d(n) \\
\hat{\boldsymbol{c}}(n+1) & =\hat{\boldsymbol{c}}(n)+\mu d^{*}(n)[z(n)-\hat{z}(n)]
\end{aligned}
$$

where $z(n)=\boldsymbol{c}(n) d(n)+\boldsymbol{\xi}(n)$ is the $L$-dimensional desired response and $\mu$ is the step size parameter. The above algorithm is equivalent to $L$ one-dimensional algorithms applied in parallel, since each element of $\hat{\boldsymbol{c}}(n)$ is updated independently of the others.

To evaluate the desired steady-state cross correlations $\boldsymbol{E}$ and $\boldsymbol{X}$, it is convenient to define $\boldsymbol{E}(n)=E\left\{\boldsymbol{\varepsilon}(n) \varepsilon^{\prime}(n)\right\}$, 
$\boldsymbol{X}(n)=E\{\boldsymbol{\varepsilon}(n) \boldsymbol{c}(n)\}$, and $\boldsymbol{Y}(n)=E\{\boldsymbol{\varepsilon}(n) \boldsymbol{c}(n+1)\}$. For the second-order model the covariance recursion is given by

$$
\begin{array}{r}
\boldsymbol{E}(n+1)=(1-\mu)^{2} \boldsymbol{E}(n)+2(1-\mu)\left[\left(f_{0}-1\right) \boldsymbol{X}(n)+f_{1} \boldsymbol{Y}(n)\right] \\
+2(1-\rho) \boldsymbol{C}+\mu^{2} N \quad(9)
\end{array}
$$

where $N$ is the covariance of the noise $\xi(n)$, and we have used the fact that both the error $\varepsilon(n)$ and the channel vectors $\boldsymbol{c}(n+1), \boldsymbol{c}(n)$ are uncorrelated with $\boldsymbol{\xi}(n)$. The quantity $\rho$ in the above expression represents the one-step channel correlation coefficient $\rho=f_{0} /\left(1-f_{1}\right)$. The recursions for $X(n)$ and $\boldsymbol{Y}(n)$ are obtained in a similar manner

$$
\begin{aligned}
& \boldsymbol{X}(n+1)=(1-\mu)\left[f_{0} \boldsymbol{X}(n)+f_{1} \boldsymbol{Y}(n)\right]+(1-\rho) \boldsymbol{C} \\
& \boldsymbol{Y}(n+1)=(1-\mu) \boldsymbol{X}(n)-(1-\rho) \boldsymbol{C} .
\end{aligned}
$$

Steady-state solutions define $\boldsymbol{E}$ and $\boldsymbol{X}$ needed for the evaluation of the error probability:

$$
\begin{gathered}
\boldsymbol{E}=\frac{1}{1-(1-\mu)^{2}}\left\{2(1-\mu)\left[f_{0}-1+f_{1}(1-\mu)\right]\right. \\
\left.\cdot \boldsymbol{X}-2(1-\rho)\left[1-f_{1}(1-\mu)\right] \boldsymbol{C}+\mu^{2} \boldsymbol{N}\right\} \\
X=(1-\rho) \frac{1-f_{1}(1-\mu)}{1-f_{0}(1-\mu)-f_{1}(1-\mu)^{2}} C .
\end{gathered}
$$

These expressions can also be used for a first-order fading model, by setting $f_{1}=0$. Unlike in the case of the Kalman filter [7], it is seen that, in general, $X \neq \boldsymbol{E}$.

A more computationally intensive solution with faster convergence is offered by an RLS algorithm whose performance is governed by the choice of the forgetting factor $\lambda$. In the given configuration, a standard RLS algorithm produces the same error with the fixed value of $\mu$ substituted by a varying step size $\mu(n)$, recursively defined by $\mu(n+1)=\mu(n) /(\lambda+\mu(n))$. Since $\mu(n)$ is independent of the channel estimation error $\varepsilon(n)$, the steady-state cross correlations $\boldsymbol{E}$ and $\boldsymbol{X}$ of the RLS estimator will equal those of the LMS estimator with $\mu=1-\lambda$. The fact that the LMS algorithm may have the same tracking capabilities as the RLS algorithm is not surprising since we are dealing with single-tap adaptive filters, for which there are no additional stability restrictions imposed on the LMS estimator due to the filter size [8]. Consequently, it is possible to choose the step size optimally while not violating the stability constraints. The optimality criterion in our case is minimization of the probability of error, which can be carried out numerically, resulting in the optimal step size as a function of the model parameters $f_{0}$ and $f_{1}$. The total performance degradation incurred by an adaptive channel estimator will therefore depend on the actual fading rate and on the deviation of the step size (or the forgetting factor) from the optimal value.

\section{NUMERICAL EXAMPLES}

We consider a mobile radio CDMA channel with total available spreading ratio of 127 . The cross-correlation matrix $\boldsymbol{R}$ is calculated from a set of flip-coin sequences with elements \pm 1 . A two-path channel is assumed for each user, with

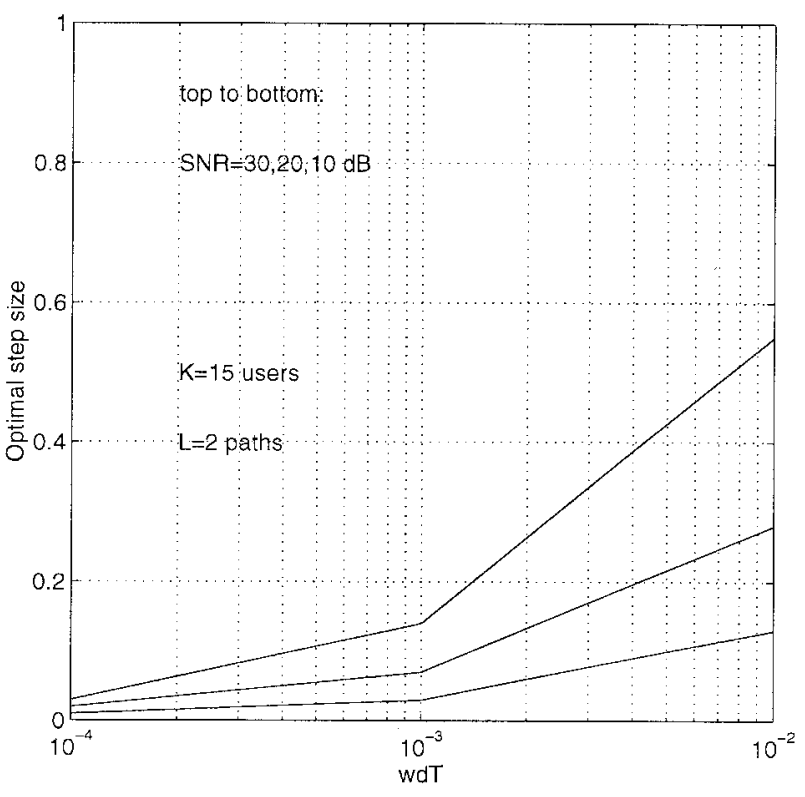

Fig. 2. Optimal step size as a function of fading rate.

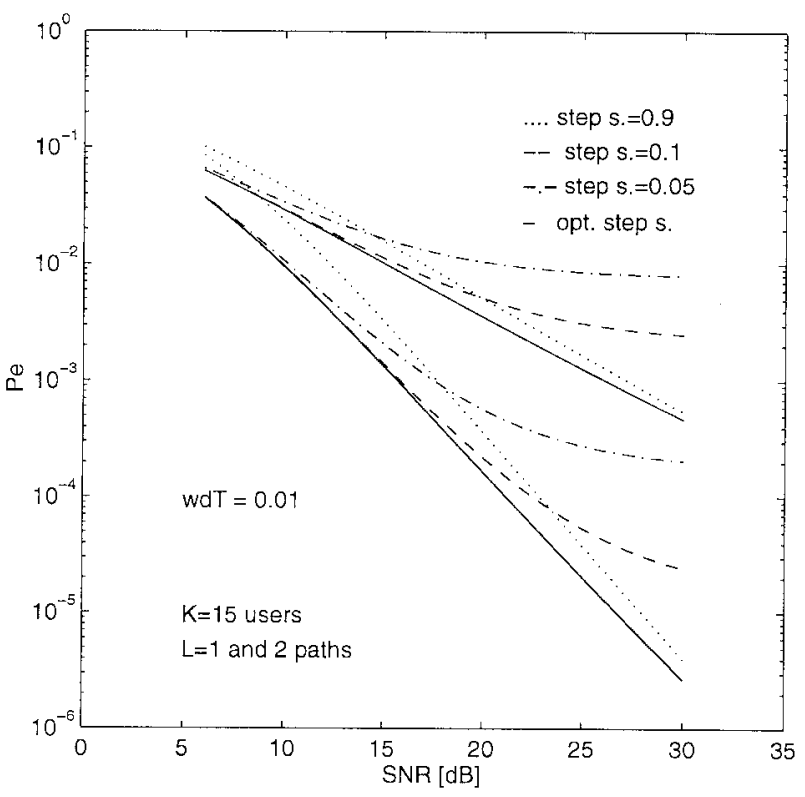

Fig. 3. Performance as a function of SNR.

independently fading paths of equal variances. We focus on a second-order model with critical damping, $\xi=1 / \sqrt{2}$, for which the Doppler spread is $\omega_{d}=\omega_{n}$.

The optimal step size as a function of the channel fading rate is summarized in Fig. 2, with signal-to-noise ration (SNR) as the parameter. It is seen that relatively large variations exist in the optimal step size with both the change in SNR and the fading rate. However, there exists a range of step sizes around the optimal value for which the performance shall not be seriously disturbed.

Performance sensitivity to the choice of the step size is examined in Fig. 3, which shows the error probability versus SNR. The fading model remains unchanged, with $\omega_{d} T$ fixed at 0.01 , corresponding to a vehicle moving at about $60 \mathrm{mi} / \mathrm{h}(100$ $\mathrm{km} / \mathrm{h}$ ), assuming a data rate of $9600 \mathrm{bits} / \mathrm{s}$ in the $900 \mathrm{MHz}$ 


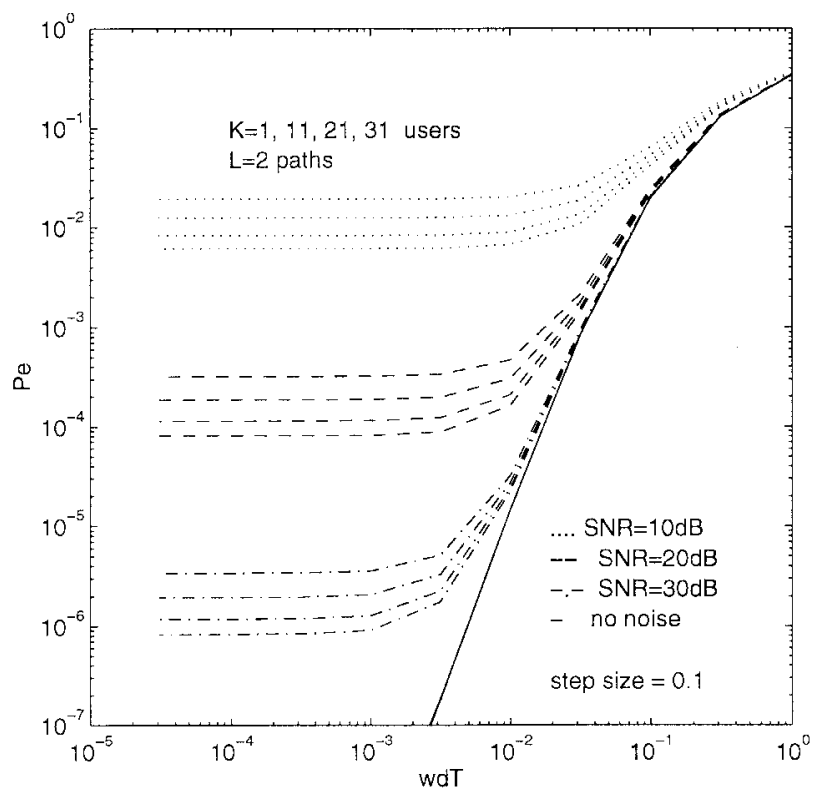

Fig. 4. Performance as a function of fading rate.

band. The reference curve is obtained by using the optimal step size for each presented value of SNR. The value $\mu=0.9$ results in the largest performance deviation at low to moderate SNR. The value $\mu=0.1$ presents a good match for the optimal case throughout the range of SNR's from 5 to $15 \mathrm{~dB}$ but results in an increasing degradation thereafter. Further decrease of the step size to 0.05 and below this value, however, may result in serious performance degradation characterized by high error floors. For a second-order fading process there will be a degradation in performance between the LMS estimator with the optimally chosen step size and the Kalman filter. For the channel parameters of Fig. 3, this degradation stays below $0.5 \mathrm{~dB}$ throughout the range of SNR's presented.

Fig. 4 shows the probability of error versus the normalized fading rate. The step size is fixed at $\mu=0.1$. The three sets of curves correspond to the SNR of 10, 20, and $30 \mathrm{~dB}$. Each set contains four curves obtained by varying the number of users. The lowest of the four error rates corresponds to the single $(K=1)$ user, while as the number of interfering users increases to 10,20, and 30, the error rate increases for the given SNR and fading rate. However, this degradation exists only for finite values of SNR. When the thermal noise vanishes, the probability of error curves corresponding to different numbers of users collapse into a single curve. This curve, labeled "no noise," represents the error probability floor as a function of fading rate. Thus, near-far resistance is preserved for all fading rates. This feature stems from the chosen structure of the receiver in which multipath decorrelation is performed first so the error floor does not depend on the number of users.

\section{REFERENCES}

[1] A. Klein and P. Baier, "Linear unbiased data estimation in mobile radio system applying CDMA," IEEE J. Select. Areas Commun., vol. 11, pp. 1058-1066, Sept. 1993.

[2] Z. Zvonar and D. Brady, "Suboptimal multiuser detector for frequency selective Rayleigh fading synchronous CDMA channels," IEEE Trans. Commun., vol. 43, pp. 154-157, Feb./Mar./Apr. 1995.

[3] M. Varanasi, "Parallel group detection for synchronous CDMA communication over frequency-selective Rayleigh fading channels," IEEE Trans. Inform. Theory, vol. 42, pp. 116-128, Jan. 1996.

[4] S. Vasudevan and M. Varanasi, "Achieving near-optimum asymptotic efficiency and fading resistance over the time-varying Rayleigh-faded CDMA channel," IEEE Trans. Commun., vol. 44, pp. 1130-1143, Sept. 1996.

[5] Z. Zvonar and M. Stojanovic, "Performance of antenna diversity multiuser receivers in CDMA channels with imperfect fading estimation," Wireless Personal Commun., vol. 3, pp. 91-110, Member, IEEE1996.

[6] H. Wu and A. Duel-Hallen, "Multiuser detection with differentially encoded data for mismatched flat Rayleigh fading CDMA channels," in Proc. 1996 Conf. Information Sciences and Systems, Princeton, NJ, Mar. 1996, pp. 332-337.

[7] M. Stojanovic and Z. Zvonar, "Linear multiuser detection in timevarying multipath fading channels," in Proc. 1996 Conf. Information Sciences and Systems, Princeton, NJ, Mar. 1996, pp. 349-354.

[8] E. Elefteriou and D. Falconer, "Tracking properties and steady-state performance of RLS adaptive filter algorithms," IEEE Trans. ASSP Mag., vol. ASSP-34, pp. 1097-1109, Oct. 1986.

[9] J. G. Proakis, Digital Communications. New York: Mc-Graw Hill, 1989.

[10] M. Juntti, "Multiuser demodulation for DS-CDMA systems in fading channels," Ph.D. dissertation, Univ. Oulu, Oulu, Finland, Sept. 1997. 